\title{
Efeito da adição de areia de fundição residual e cal a solos argilosos no módulo de resiliência
}

\author{
Luis Miguel Gutiérrez Klinsky¹, Vivian Silveira dos Santos Bardini², \\ Glauco Tulio Pessa Fabbri
}

\begin{abstract}
The effects of different contents of foundry sand (FS), in a lateritic clayey soil (LG') and a non-lateritic clayey soil (NG') and the addition of $3 \%$ of hydrated lime, on the resilient modulus $\left(M_{R}\right)$ were investigated. The cyclic triaxial test was performed on the specimens after curing periods of $0,7,28$ and 84 days. Five models were evaluated according to their precision to represent the $M_{R}$. Environmental tests were also executed on soil sand and soil sand lime mixtures. The results showed that the addition of FS reduced the modulus of LG' mixes. Nevertheless, the mixes LG' plus $20 \%$ of FS and NG' plus $40 \%$ of FS and hydrated lime showed the highest results. Additionally, was observed that hydrated lime increased the $M_{R}$, and this was more obvious at high curing periods. The environmental tests results indicate that FS reuse is not dangerous.
\end{abstract}

Keywords: Waste foundry sand. Lateritic soils. Resilient módulus.

Resumo: Neste estudo são avaliados os efeitos da adição de diferentes teores de areia de fundição residual (AFR) e de $3 \%$ de cal hidratada a dois solos argilosos, um de comportamento laterítico (LG') e um não-laterítico (NG'), à luz dos valores do módulo de resiliência $\left(M_{R}\right)$, obtido de ensaios triaxiais cíclicos para diversos tempos de cura (0, 7, 28 e 84 dias). Cinco modelos foram avaliados segundo seu desempenho para representar o $M_{R}$. Também foram realizados ensaios ambientais em misturas dos solos com AFR e com cal hidratada. Concluiu-se que a adição da AFR reduziu os valores dos módulos das misturas com LG', mas teores de 20 e $40 \%$ incrementaram o $M_{R}$ do solo NG'+Cal. Já a adição da cal aumentou os valores de $M_{R}$ das misturas e foi verificado que o tempo de cura é determinante para ganhos significativos. Os ensaios ambientais não indicaram maiores riscos com relação ao reaproveitamento do resíduo.

Palavras-chave: Areia de fundição residual. Solos lateríticos. Módulo de resiliência.

\section{INTRODUÇÃO}

Em 1986 a AASHTO (American Association of State Highway and Transportation Offici$a l s$ ), incorporou o módulo de resiliência $\left(M_{R}\right)$ como parâmetro para caracterizar os materiais geotécnicos dos pavimentos, em especial do subleito. $\mathrm{Na}$ atualidade, para qualquer procedimento de projeto ou análise mecanística, a utilização do módulo de resiliência ou $M_{R}$, dos materiais constituintes da estrutura dos pavimentos é de vital importância.

São vários os fatores que influenciam no comportamento elástico dos materiais utilizados em pavimentação, tais como: características físicas, o estado de tensão a que está sujeito, teor de umidade e grau de compactação, além de fatores climáticos. Neste estudo, o objetivo foi avaliar os efeitos da incorporação de diversos teores de areia

\footnotetext{
1 Centro de Pesquisas Rodoviárias, CCRNovaDutra, Grupo CCR. Santa Isabel, SP, Brasil. E-mail: luisgk@outlook.com

2 Departamento de Engenharia de Transportes, Escola de Engenharia de São Carlos, Universidade de São Paulo, São Carlos, SP, Brasil. E-mail: vibardini@yahoo.com.br

${ }^{3}$ Departamento de Engenharia de Transportes, Escola de Engenharia de São Carlos, Universida de de São Paulo, São Carlos, SP, Brasil. E-mail: glauco@sc.usp.br
}

Manuscrito recebido em 29/04/2013 e aprovado para publicação em 30/04/2014. Este artigo é parte de TRANSPORTES v. 22, n. 2, 2014. ISSN: 2237-1346 (online).

DOI: http://dx.doi.org/10.14295/transportes.v22i2.679 de fundição residual e também da adição de $3 \%$ cal hidratada nos valores do $M_{R}$ de dois solos argilosos, um de comportamento não laterítico e outro de comportamento laterítico segundo a MCT. Para isso, foram realizados ensaios triaxiais cíclicos em corpos de prova moldados com as misturas resultantes.

Inicialmente, foram estudados cinco modelos matemáticos para a representação dos valores dos módulos de resiliência em função do estado de tensões para, posteriormente, escolher o de melhor desempenho e, com ele, determinar valores de módulo de resiliência para cada material estudado para um determinado par de tensões fixo (de confinamento e desvio).

Finalmente, os ensaios de solubilização e lixiviação foram realizados na areia de fundição residual, nos solos LG' e NG', e em misturas contendo $60 \%$ do resíduo, com e sem a adição de cal hidratada.

\section{REVISÃO BIBLIOGRÁFICA}

As propriedades elásticas dos materiais utilizados na construção de pavimentos têm sido caracterizadas através do módulo de resiliência $\left(M_{R}\right)$. Este parâmetro é expresso como a relação 
entre a tensão desvio $\left(\sigma_{1}-\sigma_{3}\right)$ aplicada ao material e a deformação axial elástica recuperável $\left(\varepsilon_{\mathrm{r}}\right)$ após a aplicação da tensão.

O ensaio Triaxial Cíclico é utilizado para obter modelos matemáticos constitutivos que representam o $M_{R}$ em função das tensões atuantes. A literatura fornece várias equações que têm sido utilizadas para modelar o $M_{R}$ de solos e materiais granulares.

$\mathrm{O}$ modelo em função da tensão confinante $\left(\sigma_{3}\right), \quad M_{R}=k_{1} \sigma_{3}^{k_{2}}$, foi proposto por Dunlap (1963), e é utilizado principalmente para materiais granulares, onde o módulo de resiliência apresenta forte dependência da tensão de confinamento. Moosazadeh e Witczak (1981) apresentaram a relação $M_{R}=k_{1} \sigma_{d}^{k_{2}}$, que considera apenas a tensão desvio $\left(\sigma_{\mathrm{d}}\right)$ na representação do módulo de resiliência e que apresenta um desempenho regular para solos finos, principalmente os argilosos.

A NCHRP 1-28 recomenda o modelo composto $M_{R}=k_{1} \sigma_{3}^{k_{2}} \sigma_{d}^{k_{3}}$. Vários autores (Ferreira, 2002; Medina e Motta, 2005; Klinsky 2008, Solanki et al. 2010) têm relatado que essa equação, que considera a tensão desvio e a tensão confinante, produz boa previsão do módulo de resiliência, independente da granulometria do material avaliado.

O Guia de Dimensionamento MecanísticoEmpírico da AASHTO (2002) recomenda a relação $M_{R}=k_{1} p_{a}\left(\frac{\theta}{p_{a}}\right)^{k_{2}}\left(\frac{\tau_{o c t}}{p_{a}}+1\right)^{k_{3}}$, para a representação do módulo de resiliência, onde $\theta$ é a soma das três tensões principais $\left(\sigma_{1}, \sigma_{2}, \sigma_{3}\right), \tau_{\text {oct }}$ é a tensão octaédrica cisalhante atuante no material e $p_{a}$ é a pressão atmosférica.

Andrei et al. (2004) avaliaram o protocolo usualmente utilizado para determinação do módulo de resiliência nos Estados Unidos (NCHRP 128). Os autores observaram que o modelo $M_{R}=k_{1} p_{a} k_{2}^{\left(\frac{\sigma_{3}}{p_{a}}\right)} k_{3}^{\left(\frac{\sigma_{d}}{p_{a}}\right)}$ apresentou um bom ajuste com relação aos dados obtidos nos solos estudados. A vantagem desse modelo é que as tensões $\sigma_{3}=0$ ou $\sigma_{d}=0$ podem ser utilizadas para calcular o valor de módulo de resiliência.

Com relação à adição de cal hidratada a solos, pode se afirmar que essa metodologia é muito utilizada no âmbito rodoviário para aproveitar materiais que seriam considerados inadequados para seu emprego na construção de camadas de pavimentos. A estabilização de solos com cal hi- dratada, basicamente, é um método físicoquímico que tem o propósito de melhorar as características mecânicas, aumentar a resistência à ação da água e incrementar o valor de suporte do solo, (Baptista, 1976).

A adição de cal hidratada a solos reativos melhora substancialmente a resistência dos solos através das reações pozolânicas, que sucedem em longos períodos de tempo. Geralmente, esse incremento na resistência acontece em concomitância com um enrijecimento do material, mudando sua relação tensão-deformação. De acordo com Little (1999), as misturas solo-cal apresentam maior resistência às tensões desviatórias e, por outra parte, exibem menores deformações. Como resultado, a rigidez de solos estabilizados com cal hidratada é de 10 a 25 vezes maior que a dos solos naturais. Consequentemente, esses materiais auxiliam na redução da deformação total do pavimento onde forem utilizados.

As características mecânicas de um solo laterítico estabilizado com cal foram estudadas por Lovato (2004). Nesse estudo, o autor investigou o efeito da adição de cal calcítica e cal dolomítica (em teores crescentes: $3 \%, 4 \%$ e 5\%), nas propriedades resilientes de um solo laterítico, com o objetivo de utilizar os materiais resultantes em camadas de pavimentos semirrígidos. Os valores de $M_{R}$ obtidos com a adição $3 \%$ de cal calcítica após 56 dias de cura foram superiores a $5.000 \mathrm{MPa}$; já a adição de $3 \%$ de cal dolomítica produziu valores próximos a $1600 \mathrm{MPa}$.

Solanki et al. (2010) avaliaram o efeito da incorporação de cal (3, 6 e 9\%) nas propriedades resilientes de quatro solos argilosos. Os solos no estado natural apresentaram $M_{R}$ próximos a 100 MPa. A adição de $3 \%$ de cal aumentou o $M_{R}$ dos quatro solos até valores em torno de $700 \mathrm{MPa}$. Entretanto, a adição de $6 \%$ e $9 \%$ de cal não proporcionou incrementos dos módulos de resiliência proporcionais à quantidade adicionada e os valores obtidos, para esses teores, foram semelhantes aos com 3,0\% de cal.

Alguns estudos de estradas construídas com misturas de solo-cal, mostram valores de $M_{R}$ entre $210 \mathrm{MPa}$ a $3.500 \mathrm{MPa}$, obtidos através de retroanálise, (Little, 1999). Materiais geotécnicos com esses níveis de $M_{R}$ melhoram consideravelmente a distribuição de tensões no pavimento, contudo, os módulos não são tão elevados para originar tensões de tração nas camadas de base de pavimentos de volume de tráfego intenso. 
Jung et al. (2008) avaliaram também, através do FWD (Falling Weight Deflectometer), sete rodovias construídas com bases, sub-bases ou subleitos estabilizados com cal. Os pavimentos avaliados por esses autores estão localizados no Estado de Indiana (EUA) e tinham de 5 a 10 anos de serviço na época em que foram estudados. Os resultados da retroanálise comprovaram um incremento do $M_{R}$ dos solos estabilizados de 4 a 11 vezes, quando comparado com os valores encontrados nos solos naturais. Desse modo, os autores concluem que a adição de cal aumenta consideravelmente a rigidez dos solos, o que poderia resultar em projetos de pavimentos com espessuras inferiores.

\section{MATERIAIS E MÉTODOS}

Foram coletados dois solos argilosos próximos da cidade de Piracicaba, Estado de São Paulo. Um solo foi classificado, segundo a metodologia MCT (Miniatura, Compactado, Tropical), como Laterítico Argiloso (LG') e o outro como Não Laterítico Argiloso (NG'). Outras características destes solos são apresentadas na Tabela 1 e na Figura 1.

A cal hidratada empregada neste estudo foi do tipo CH-I. A areia de fundição residual (AFR) foi coletada na Siderúrgica DEDINI, localizada na cidade de Piracicaba/SP. Esta empresa utiliza a técnica de areia de macho com adição de resina furânica para compor os moldes de fundição. A Figura 1 apresenta as distribuições granulométricas da AFR e dos dois solos argilosos.

Nesta pesquisa foram compostos quatro conjuntos de misturas de solo, areia e cal, a saber:

I) Solo LG' + AFR;

II) Solo NG' + AFR;

III) Solo LG' + AFR + Cal;

IV) Solo NG' + AFR + Cal.

Para os quatro conjuntos de misturas foram utilizados cinco teores de AFR: 0, 20, 40, 60 e $70 \%$. Já o teor de cal foi fixado em $3 \%$, com o objetivo de melhorar as propriedades mecânicas das misturas, como recomendam Oliveira (2005) e Leandro (2005).

$\mathrm{O}$ ensaio triaxial cíclico foi realizado em corpos de prova (CP) de $200 \mathrm{~mm}$ de altura e 100 mm de diâmetro, segundo a AASHTO T 307-99, utilizando-se os níveis de tensões recomendados para camadas de bases e sub-bases de pavimentos. Os CP foram compactados estaticamente, em cinco camadas, objetivando reproduzir os parâmetros de compactação obtidos no ensaio Proctor realizado na energia intermediária. A umidade ótima ( $\left.\mathrm{W}_{\text {ot }} \pm 0,3 \%\right)$ e a massa específica seca máxima (grau de compactação $\mathrm{GC}=100 \pm 2 \%$ ) foram os parâmetros empregados para aceitar ou descartar os CP durante a compactação.

\begin{tabular}{|c|c|c|}
\hline Solo & $\begin{array}{l}\text { Laterítico Argiloso } \\
\text { (LG') }\end{array}$ & $\begin{array}{c}\text { Não Laterítico Argiloso } \\
\text { (NG') }\end{array}$ \\
\hline Massa Específica dos Sólidos $\left(\mathrm{g} / \mathrm{cm}^{3}\right)$ & 2,745 & 2,825 \\
\hline LL (\%) & 58 & 46 \\
\hline LP(\%) & 34 & 33 \\
\hline $\mathrm{IP}(\%)$ & 24 & 13 \\
\hline Passante 4,80 mm (\%) & 100 & 100 \\
\hline Passante 2,00 mm (\%) & 100 & 100 \\
\hline Passante 0,42 mm (\%) & 100 & 100 \\
\hline Passante 0,074 mm (\%) & 96 & 85 \\
\hline Passante 0,005 mm (\%) & 64 & 49 \\
\hline Classificação HRB & $A-7-5$ & $A-7-5$ \\
\hline Classificação USCS & $\mathrm{MH}$ & ML \\
\hline CBR após 96h imerso (\%) & 35 & 5 \\
\hline Expansão (\%) & 0,19 & 5,90 \\
\hline mini-CBR após 24h imerso (\%) & 25 & 6 \\
\hline Contração (\%) & 1,30 & 1,70 \\
\hline
\end{tabular}




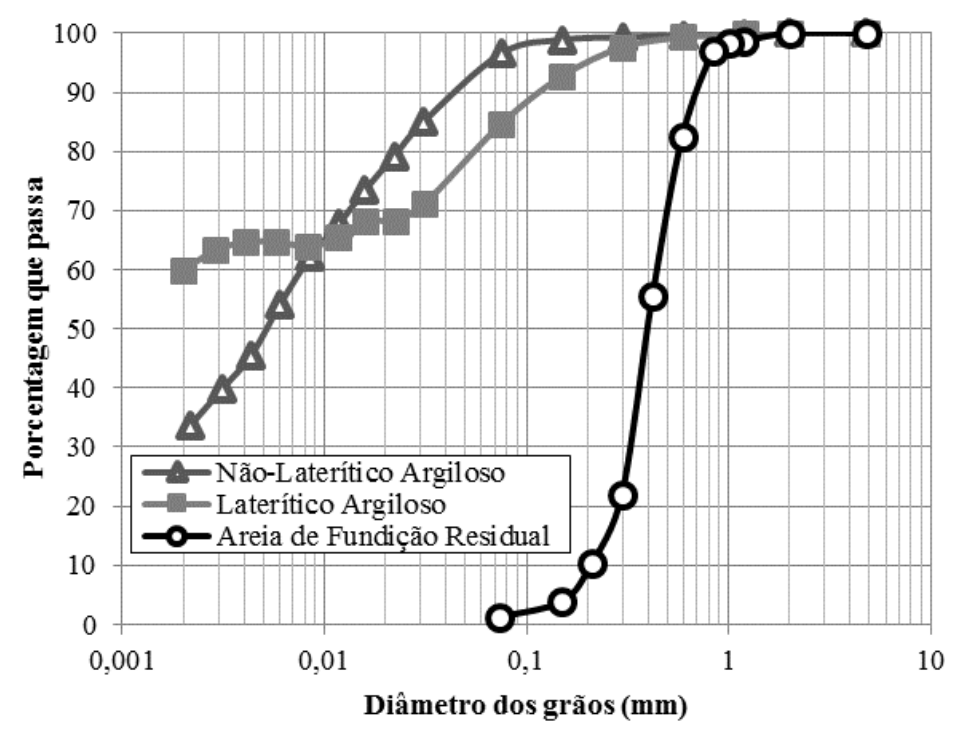

Figura 1 - Curvas granulométricas dos materiais utilizados

Para cada mistura dos quatro conjuntos foram compactados dois corpos-de-prova e, cada $\mathrm{CP}$ foi ensaiado no mínimo duas vezes. Segundo diversas publicações (Pan et al. 2006; Karasahin et al. 1993), o ensaio triaxial cíclico pode ser executado repetidas vezes no mesmo $\mathrm{CP}$ por aplicar tensões que solicitam o material apenas na faixa elástica.

Tanto no conjunto Solo+AFR como no conjunto Solo+AFR+Cal, foram estudados quatro tempos de cura: 0, 7, 28 e 84 dias após compactação dos corpos-de-prova, para avaliar o efeito do tempo de cura nos valores dos módulos de resiliência das misturas.

\section{APRESENTAÇÃO E ANÁLISE DOS RESULTADOS}

\subsection{Resultados do Módulo de Resiliência}

Inicialmente foram utilizados cinco modelos matemáticos, apresentados na Tabela 2, para a representação do módulo de resiliência das misturas. O coeficiente de determinação $\mathrm{R}^{2}$ foi o critério utilizado para avaliar os modelos, já que este parâmetro é adequado para estimar a acurácia do ajuste estatístico alcançado pelo modelo utilizado. Segundo o Guia de Dimensionamento de Pavimentos da AASHTO (2002), valores de $\mathrm{R}^{2}$ superiores a 0,90 indicam que as equações são adequadas para representar o módulo de resiliência.

Assim, para cada modelo estudado, foi determinada a porcentagem de ensaios que apresentaram bom desempenho na previsão do módulo de resiliência, em função do número de ensaios realizados e do número de modelos que apresentam $\mathrm{R}^{2} \geq 0,90$. Na Figura 2 são apresentados estes resultados, onde nota-se que o Modelo Composto (modelo 3) é o que apresenta maior porcentagem de ensaios com $\mathrm{R}^{2} \geq 0,90$. Por outro lado, o modelo com menor porcentagem foi aquele em função da tensão confinante (modelo 2), devido ao caráter argiloso dos materiais estudados.

Tabela 2 - Modelos matemáticos avaliados para representar o módulo de resiliência

\begin{tabular}{ccc}
\hline Modelo & Nome & Equação \\
\hline 1 & Modelo em Função da Tensão Desvio & $M_{R}=k_{1} \sigma_{d}^{k_{2}}$ \\
\hline $\mathbf{2}$ & Modelo em Função da Tensão Confinante & $M_{R}=k_{1} \sigma_{3}^{k_{2}}$ \\
\hline $\mathbf{3}$ & Modelo Composto & $M_{R}=k_{1} \sigma_{3}^{k_{2}} \sigma_{d}^{k_{3}}$ \\
\hline 5 & Modelo AASHTO (2002) & $M_{R}=k_{1} p_{a}\left(\frac{\theta}{p_{a}}\right)^{k_{2}}\left(\frac{\tau_{\text {oct }}}{p_{a}}+1\right)^{k_{3}}$ \\
\hline
\end{tabular}




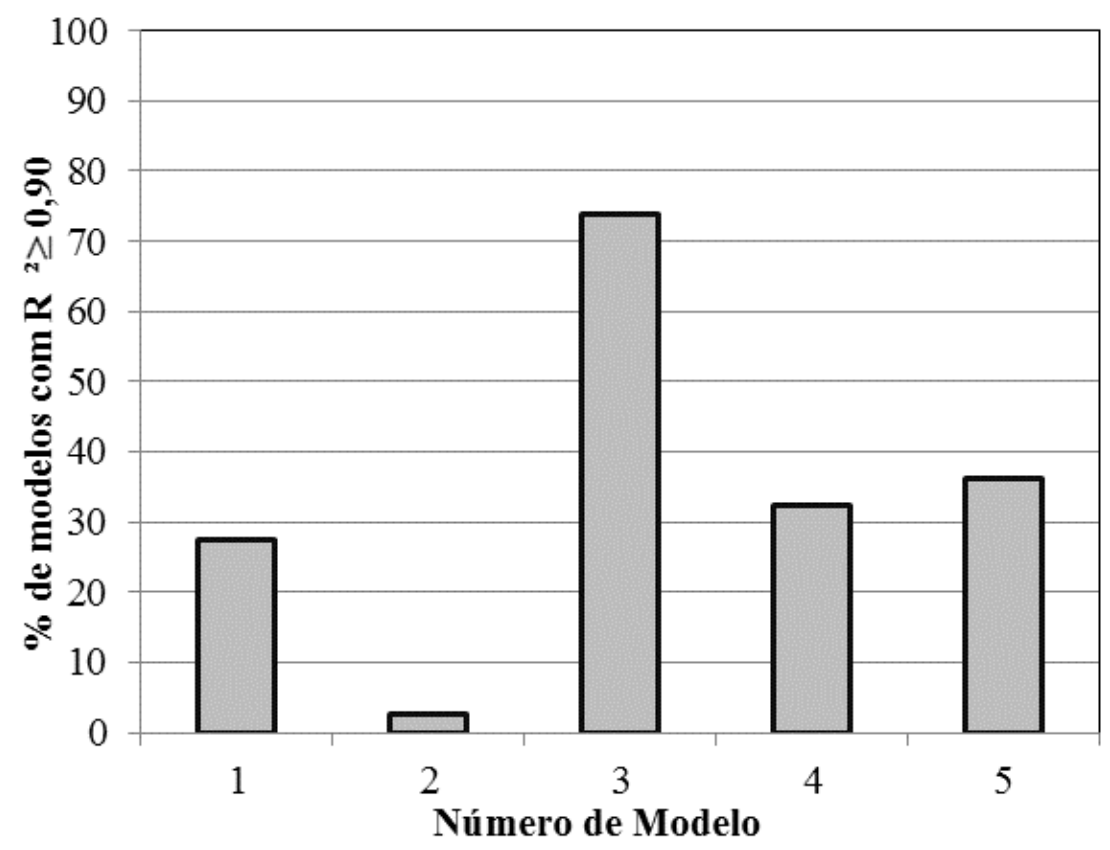

Figura 2 - Porcentagem de ensaios que apresentam $R^{2} \geq 0,90$ para cada modelo

Com relação ao Modelo da AASHTO (modelo 4) e de Andrei et al. (2004) (modelo 5), notou-se que ambos apresentam uma porcentagem de ensaios com $\mathrm{R}^{2} \geq 0,90$ muito próxima, $33 \%$ e $37 \%$, respectivamente. Não era esperado um desempenho tão ruim do Modelo da AASHTO, contudo, outros estudos (Solanki et al., 2010; Andrei et al., 2004) também relatam o desempenho pobre e inconsistente deste na representação do módulo de resiliência.

Nas Tabelas 3 e 4 são apresentados os parâmetros $\left(\mathrm{k}_{1}, \mathrm{k}_{2}, \mathrm{k}_{3}\right)$ do Modelo Composto para as misturas compostas com o solo LG' e NG', respectivamente. Vale notar que para o ajuste dos modelos foram considerados no mínimo 11 dos 15 pares de tensão utilizados no ensaio triaxial cíclico, como recomenda a AASHTO T 307-99.

As massas específicas secas máximas $(\gamma \mathrm{d})$ e as umidades ótimas ( $\mathrm{W}_{\mathrm{ot}}$ ) obtidas na compactação, utilizadas na compactação dos corpos de prova utilizados no ensaio triaxial cíclico, também são apresentadas também nas Tabelas 3 e 4, assim como os valores de Módulo de Resiliência $\left(M_{R}\right)$ calculados a partir do Modelo Composto e do último par de tensões utilizado no ensaio triaxial cíclico: $\sigma_{3}=137,9 \mathrm{kPa}$ e $\sigma_{\mathrm{d}}=275,8 \mathrm{kPa}$.

Nota-se, nas Tabelas 3 e 4, que os parâmetros resultantes do modelo são bastante variáveis; contudo, segundo Boudreau e Wang (2003), não existe referência que recomende valores máximos ou mínimos. Vale notar que $\mathrm{k}_{1}, \mathrm{k}_{2}, \mathrm{k}_{3}$ são apenas parâmetros de ajuste do modelo com base nos valores de $M_{R}$ obtidos no ensaio triaxial cíclico e não representam nenhuma propriedade física ou mecânica do material.

As Figuras 3 e 4 apresentam os efeitos, nos valores do $M_{R}$, das adições de areia de fundição residual (AFR) e cal hidratada nos solos argilosos LG' e NG', respectivamente.

Das Figuras 3 e 4 pode-se extrair os efeitos em relação ao tempo de cura, à adição de AFR e à adição de cal hidratada aos solos argilosos:

\subsection{Tempo de Cura}

Aos 7 dias de cura notou-se, de maneira geral, pouco ou nenhum incremento do valor de $M_{R}$, nas misturas sem a adição de cal hidratada, como pode-se observar nas Figuras 3a e 4a. Entretanto, aos 28 e 84 dias de cura houve um incremento do módulo nas misturas com até $40 \%$ de areia de fundição residual.

Nota-se na Figura 3 a que os solos $\mathrm{LG}^{\prime}+0 \%$ e LG' $+20 \%$ apresentam MR bastante elevados, da ordem de $2.855 \mathrm{MPa}$ e $1.998 \mathrm{MPa}$, respectivamente. Vale notar que estes valores foram obtidos de modelos considerados regulares, com $\mathrm{R}^{2}=$ 0,77 (Tabela 3).

Nas misturas com a adição de 3\% de cal notou-se um claro incremento do valor de $M_{R}$ com o incremento do tempo de cura. As misturas compostas pelo solo NG' mostraram aumentos maiores de módulo aos 28 e 84 dias. 
Tabela 3 - Parâmetros do Modelo Composto e $M_{R}\left(\sigma_{3}=137,9 \mathrm{kPa}\right.$ e $\left.\sigma_{\mathrm{d}}=275,8 \mathrm{kPa}\right)$ das misturas com solo LG'

\begin{tabular}{|c|c|c|c|c|c|c|c|c|}
\hline \multirow[t]{2}{*}{ Mistura } & \multirow{2}{*}{$\begin{array}{l}\mathrm{W}_{\mathrm{ot}} \\
(\%)\end{array}$} & \multirow{2}{*}{$\gamma_{d}\left(\mathrm{~g} / \mathrm{cm}^{3}\right)$} & \multirow{2}{*}{$\begin{array}{c}\text { Cura } \\
\text { (dias) }\end{array}$} & \multicolumn{4}{|c|}{ Modelo Composto } & \multirow[t]{2}{*}{$\begin{array}{c}\mathrm{MR} \\
(\mathrm{MPa})\end{array}$} \\
\hline & & & & $\mathrm{k} 1$ & k2 & k3 & $\mathrm{R}^{2}$ & \\
\hline $\mathrm{LG}^{\prime}+0 \%$ & 20,5 & 1,760 & 0 & $15.441,6$ & $-0,27$ & $-0,30$ & 0,90 & 765 \\
\hline$L G^{\prime}+20 \%$ & 16,8 & 1,905 & 0 & $11.191,7$ & $-0,11$ & $-0,35$ & 0,90 & 890 \\
\hline$L^{\prime}+40 \%$ & 14,0 & 2,010 & 0 & $1.561,7$ & 0,25 & $-0,65$ & 0,92 & 139 \\
\hline$L^{\prime}+60 \%$ & 11,1 & 2,100 & 0 & 105,8 & 0,91 & $-0,67$ & 0,92 & 223 \\
\hline $\mathrm{LG}^{\prime}+70 \%$ & 10,0 & 2,100 & 0 & 37,5 & 0,91 & $-0,45$ & 0,96 & 263 \\
\hline $\mathrm{LG}^{\prime}+0 \%$ & 20,5 & 1,760 & 7 & $6.039,4$ & 0,04 & $-0,39$ & 0,90 & 842 \\
\hline$L G^{\prime}+20 \%$ & 16,8 & 1,905 & 7 & $24.812,8$ & $-0,52$ & $-0,13$ & 0,85 & 920 \\
\hline$L^{\prime}+40 \%$ & 14,0 & 2,010 & 7 & $1.950,8$ & 0,10 & $-0,46$ & 0,93 & 244 \\
\hline$L^{\prime}+60 \%$ & 11,1 & 2,100 & 7 & 90,1 & 0,78 & $-0,55$ & 0,93 & 185 \\
\hline $\mathrm{LG}^{\prime}+70 \%$ & 10,0 & 2,100 & 7 & 6,1 & 1,12 & $-0,38$ & 0,93 & 176 \\
\hline $\mathrm{LG}^{\prime}+0 \%$ & 20,5 & 1,760 & 28 & $102.625,1$ & $-0,15$ & $-0,70$ & 0,97 & 957 \\
\hline$L^{\prime}+20 \%$ & 16,8 & 1,905 & 28 & $339.763,1$ & $-0,56$ & $-0,53$ & 0,93 & 1.064 \\
\hline $\mathrm{LG}^{\prime}+40 \%$ & 14,0 & 2,010 & 28 & $7.871,8$ & 0,03 & $-0,69$ & 0,91 & 189 \\
\hline$L^{\prime}+60 \%$ & 11,1 & 2,100 & 28 & $1.196,6$ & 0,48 & $-0,71$ & 0,94 & 238 \\
\hline $\mathrm{LG}^{\prime}+70 \%$ & 10,0 & 2,100 & 28 & 334,3 & 0,44 & $-0,43$ & 0,94 & 267 \\
\hline $\mathrm{LG}^{\prime}+0 \%$ & 20,5 & 1,760 & 84 & $18.944,6$ & 0,07 & $-0,40$ & 0,77 & 2.855 \\
\hline$L^{\prime}+20 \%$ & 16,8 & 1,905 & 84 & $575.647,8$ & $-0,83$ & $-0,28$ & 0,77 & 1.998 \\
\hline $\mathrm{LG}^{\prime}+40 \%$ & 14,0 & 2,010 & 84 & $91.847,9$ & $-0,08$ & $-0,82$ & 0,92 & 598 \\
\hline$L^{\prime}+60 \%$ & 11,1 & 2,100 & 84 & $293.196,4$ & $-0,08$ & $-1,21$ & 0,93 & 226 \\
\hline $\mathrm{LG}^{\prime}+70 \%$ & 10,0 & 2,100 & 84 & 164,3 & 1,03 & $-0,87$ & 0,91 & 198 \\
\hline $\mathrm{LG}^{\prime}+0 \%+\mathrm{Cal}$ & 22,0 & 1,725 & 0 & $1.447 .998,6$ & $-0,06$ & $-1,20$ & 0,82 & 1.281 \\
\hline $\mathrm{LG}^{\prime}+20 \%+\mathrm{Cal}$ & 18,7 & 1,820 & 0 & $63.731,0$ & 0,17 & $-0,84$ & 0,94 & 1.298 \\
\hline $\mathrm{LG}^{\prime}+40 \%+\mathrm{Cal}$ & 16,2 & 1,910 & 0 & $13.769,8$ & $-0,07$ & $-0,58$ & 0,92 & 376 \\
\hline $\mathrm{LG}^{\prime}+60 \%+\mathrm{Cal}$ & 12,5 & 2,000 & 0 & $21.076,6$ & 0,02 & $-0,78$ & 0,91 & 293 \\
\hline $\mathrm{LG}^{\prime}+70 \%+\mathrm{Cal}$ & 11,3 & 2,055 & 0 & $3.596,2$ & 0,40 & $-0,83$ & 0,91 & 248 \\
\hline $\mathrm{LG}^{\prime}+0 \%+\mathrm{Cal}$ & 22,0 & 1,725 & 7 & $386.993,6$ & $-0,43$ & $-0,56$ & 0,91 & 1.977 \\
\hline $\mathrm{LG}^{\prime}+20 \%+\mathrm{Cal}$ & 18,7 & 1,820 & 7 & $60.303,4$ & $-0,57$ & $-0,22$ & 0,93 & 1.068 \\
\hline $\mathrm{LG}^{\prime}+40 \%+\mathrm{Cal}$ & 16,2 & 1,910 & 7 & $9.275,4$ & $-0,08$ & $-0,51$ & 0,94 & 349 \\
\hline $\mathrm{LG}^{\prime}+60 \%+\mathrm{Cal}$ & 12,5 & 2,000 & 7 & $26.000,3$ & 0,33 & $-0,96$ & 0,90 & 605 \\
\hline $\mathrm{LG}^{\prime}+70 \%+\mathrm{Cal}$ & 11,3 & 2,055 & 7 & $3.900,5$ & 0,27 & $-0,67$ & 0,89 & 350 \\
\hline $\mathrm{LG}^{\prime}+0 \%+\mathrm{Cal}$ & 22,0 & 1,725 & 28 & $13.948,8$ & 0,75 & $-0,96$ & 0,85 & 2.522 \\
\hline $\mathrm{LG}^{\prime}+20 \%+\mathrm{Cal}$ & 18,7 & 1,820 & 28 & $3.578 .428,6$ & $-0,75$ & $-0,71$ & 0,85 & 1.657 \\
\hline $\mathrm{LG}^{\prime}+40 \%+\mathrm{Cal}$ & 16,2 & 1,910 & 28 & $16.348,1$ & 0,08 & $-0,63$ & 0,93 & 694 \\
\hline $\mathrm{LG}^{\prime}+60 \%+\mathrm{Cal}$ & 12,5 & 2,000 & 28 & $870.428,4$ & $-0,37$ & $-0,87$ & 0,92 & 1.026 \\
\hline $\mathrm{LG}^{\prime}+70 \%+\mathrm{Cal}$ & 11,3 & 2,055 & 28 & $5.866,5$ & 0,04 & $-0,42$ & 0,93 & 666 \\
\hline $\mathrm{LG}^{\prime}+0 \%+\mathrm{Cal}$ & 22,0 & 1,725 & 84 & $54.386,6$ & $-0,39$ & $-0,20$ & 0,74 & 2.640 \\
\hline $\mathrm{LG}^{\prime}+20 \%+\mathrm{Cal}$ & 18,7 & 1,820 & 84 & $708.690,0$ & $-0,09$ & $-0,98$ & 0,86 & 1.841 \\
\hline $\mathrm{LG}^{\prime}+40 \%+\mathrm{Cal}$ & 16,2 & 1,910 & 84 & $44.527 .190,2$ & $-0,66$ & $-1,26$ & 0,82 & 1.427 \\
\hline $\mathrm{LG}^{\prime}+60 \%+\mathrm{Cal}$ & 12,5 & 2,000 & 84 & $224.049,8$ & 0,13 & $-1,01$ & 0,90 & 1.436 \\
\hline $\mathrm{LG}^{\prime}+70 \%+\mathrm{Cal}$ & 11,3 & 2,055 & 84 & $281.397,9$ & 0,24 & $-1,23$ & 0,86 & 887 \\
\hline
\end{tabular}

Tabela 4 - Parâmetros do Modelo Composto e $M_{R}\left(\sigma_{3}=137,9 \mathrm{kPa}\right.$ e $\left.\sigma_{\mathrm{d}}=275,8 \mathrm{kPa}\right)$ das misturas com solo NG' (continua)

\begin{tabular}{|c|c|c|c|c|c|c|c|c|}
\hline \multirow{2}{*}{ Mistura } & \multirow{2}{*}{$\begin{array}{l}W_{o t} \\
(\%)\end{array}$} & \multirow{2}{*}{$\begin{array}{c}\gamma_{\mathrm{d}} \\
\left(\mathrm{g} / \mathrm{cm}^{3}\right)\end{array}$} & \multirow{2}{*}{$\begin{array}{l}\text { Cura } \\
\text { (dias) }\end{array}$} & \multicolumn{4}{|c|}{ Modelo Composto } & \multirow[t]{2}{*}{ MR (MPa) } \\
\hline & & & & $\mathrm{k} 1$ & k2 & k3 & $\mathrm{R}^{2}$ & \\
\hline$N G^{\prime}+0 \%$ & 21,7 & 1,625 & 0 & $6.334,6$ & $-0,06$ & $-0,53$ & 0,94 & 246 \\
\hline$N G^{\prime}+20 \%$ & 16,8 & 1,790 & 0 & $11.058,8$ & $-0,03$ & $-0,57$ & 0,95 & 391 \\
\hline$N^{\prime}+40 \%$ & 14,4 & 1,890 & 0 & $5.175,0$ & $-0,04$ & $-0,56$ & 0,97 & 191 \\
\hline$N^{\prime}+60 \%$ & 11,3 & 2,000 & 0 & $2.572,9$ & 0,14 & $-0,62$ & 0,91 & 153 \\
\hline $\mathrm{NG}^{\prime}+70 \%$ & 10,8 & 2,100 & 0 & 91,9 & 0,69 & $-0,50$ & 0,91 & 169 \\
\hline$N^{\prime}+0 \%$ & 21,7 & 1,625 & 7 & $1.822,1$ & 0,19 & $-0,52$ & 0,95 & 257 \\
\hline$N G^{\prime}+20 \%$ & 16,8 & 1,790 & 7 & $48.957,4$ & $-0,02$ & $-0,82$ & 0,94 & 440 \\
\hline$N^{\prime}+40 \%$ & 14,4 & 1,890 & 7 & $11.902,7$ & $-0,11$ & $-0,65$ & 0,97 & 184 \\
\hline$N^{\prime}+60 \%$ & 11,3 & 2,000 & 7 & $1.613,5$ & 0,16 & $-0,62$ & 0,92 & 115 \\
\hline$N^{\prime}+70 \%$ & 10,8 & 2,100 & 7 & 29,8 & 1,05 & $-0,64$ & 0,92 & 143 \\
\hline
\end{tabular}


Tabela 4 - Parâmetros do Modelo Composto e $M_{R}\left(\sigma_{3}=137,9 \mathrm{kPa}\right.$ e $\left.\sigma_{\mathrm{d}}=275,8 \mathrm{kPa}\right)$ das misturas com solo NG' (conclusão)

\begin{tabular}{|c|c|c|c|c|c|c|c|c|}
\hline \multirow{2}{*}{ Mistura } & \multirow{2}{*}{$\begin{array}{l}W_{o t} \\
(\%)\end{array}$} & \multirow{2}{*}{$\begin{array}{c}\gamma_{d} \\
\left(\mathrm{~g} / \mathrm{cm}^{3}\right)\end{array}$} & \multirow{2}{*}{$\begin{array}{c}\text { Cura } \\
\text { (dias) }\end{array}$} & \multicolumn{4}{|c|}{ Modelo Composto } & \multirow[t]{2}{*}{$\mathrm{MR}(\mathrm{MPa})$} \\
\hline & & & & k1 & k2 & k3 & $\mathrm{R}^{2}$ & \\
\hline $\mathrm{NG}^{\prime}+0 \%$ & 21,7 & 1,625 & 28 & $3.387,9$ & 0,01 & $-0,38$ & 0,96 & 409 \\
\hline$N G^{\prime}+20 \%$ & 16,8 & 1,790 & 28 & $8.609,6$ & 0,05 & $-0,55$ & 0,94 & 495 \\
\hline$N G^{\prime}+40 \%$ & 14,4 & 1,890 & 28 & $8.382,2$ & $-0,17$ & $-0,49$ & 0,93 & 229 \\
\hline$N G^{\prime}+60 \%$ & 11,3 & 2,000 & 28 & $1.632,6$ & 0,21 & $-0,66$ & 0,96 & 112 \\
\hline$N G^{\prime}+70 \%$ & 10,8 & 2,100 & 28 & 36,0 & 0,82 & $-0,51$ & 0,93 & 113 \\
\hline$N G^{\prime}+0 \%$ & 21,7 & 1,625 & 84 & $203.728,0$ & $-0,29$ & $-0,86$ & 0,95 & 372 \\
\hline$N G^{\prime}+20 \%$ & 16,8 & 1,790 & 84 & $1.017 .579,3$ & $-0,27$ & $-1,10$ & 0,90 & 572 \\
\hline$N G^{\prime}+40 \%$ & 14,4 & 1,890 & 84 & $64.728,4$ & $-0,10$ & $-0,80$ & 0,88 & 453 \\
\hline$N G^{\prime}+60 \%$ & 11,3 & 2,000 & 84 & $2.831,1$ & 0,31 & $-0,85$ & 0,94 & 108 \\
\hline $\mathrm{NG}^{\prime}+70 \%$ & 10,8 & 2,100 & 84 & 402,7 & 0,71 & $-0,86$ & 0,90 & 102 \\
\hline $\mathrm{NG}^{\prime}+0 \%+\mathrm{Cal}$ & 24,0 & 1,560 & 0 & $23.789,5$ & $-0,47$ & $-0,23$ & 0,96 & 660 \\
\hline $\mathrm{NG}^{\prime}+20 \%+\mathrm{Cal}$ & 18,7 & 1,700 & 0 & $3.656,1$ & 0,10 & $-0,40$ & 0,88 & 641 \\
\hline $\mathrm{NG}^{\prime}+40 \%+\mathrm{Cal}$ & 14,2 & 1,870 & 0 & $41.376,9$ & 0,01 & $-0,75$ & 0,95 & 649 \\
\hline $\mathrm{NG}^{\prime}+60 \%+\mathrm{Cal}$ & 13,3 & 1,975 & 0 & $2.301,0$ & 0,11 & $-0,53$ & 0,90 & 198 \\
\hline $\mathrm{NG}^{\prime}+70 \%+\mathrm{Cal}$ & 12,4 & 2,030 & 0 & 742,9 & 0,36 & $-0,62$ & 0,91 & 138 \\
\hline $\mathrm{NG}^{\prime}+0 \%+\mathrm{Cal}$ & 24,0 & 1,560 & 7 & $78.669,4$ & $-0,63$ & $-0,19$ & 0,87 & 1.180 \\
\hline $\mathrm{NG}^{\prime}+20 \%+\mathrm{Cal}$ & 18,7 & 1,700 & 7 & $149.418,3$ & $-0,26$ & $-0,59$ & 0,90 & 1.565 \\
\hline $\mathrm{NG}^{\prime}+40 \%+\mathrm{Cal}$ & 14,2 & 1,870 & 7 & $22.188,6$ & 0,38 & $-0,79$ & 0,65 & 1.656 \\
\hline $\mathrm{NG}^{\prime}+60 \%+\mathrm{Cal}$ & 13,3 & 1,975 & 7 & $18.186,5$ & 0,07 & $-0,69$ & 0,97 & 525 \\
\hline $\mathrm{NG}^{\prime}+70 \%+\mathrm{Cal}$ & 12,4 & 2,030 & 7 & $4.762,0$ & 0,05 & $-0,45$ & 0,93 & 477 \\
\hline $\mathrm{NG}^{\prime}+0 \%+\mathrm{Cal}$ & 24,0 & 1,560 & 28 & $17.661,3$ & $-0,21$ & $-0,28$ & 0,94 & 1.350 \\
\hline $\mathrm{NG}^{\prime}+20 \%+\mathrm{Cal}$ & 18,7 & 1,700 & 28 & $74.413,8$ & 0,23 & $-0,81$ & 0,93 & 2.498 \\
\hline $\mathrm{NG}^{\prime}+40 \%+\mathrm{Cal}$ & 14,2 & 1,870 & 28 & $82.123,9$ & $-0,19$ & $-0,41$ & 0,85 & 3.113 \\
\hline $\mathrm{NG}^{\prime}+60 \%+\mathrm{Cal}$ & 13,3 & 1,975 & 28 & $106.635,3$ & 0,53 & $-1,27$ & 0,86 & 1.215 \\
\hline $\mathrm{NG}^{\prime}+70 \%+\mathrm{Cal}$ & 12,4 & 2,030 & 28 & $3.120,4$ & 0,47 & $-0,53$ & 0,90 & 1.617 \\
\hline $\mathrm{NG}^{\prime}+0 \%+\mathrm{Cal}$ & 24,0 & 1,560 & 84 & $11.758,1$ & $-0,09$ & $-0,31$ & 0,77 & 1.309 \\
\hline $\mathrm{NG}^{\prime}+20 \%+\mathrm{Cal}$ & 18,7 & 1,700 & 84 & $8.674,5$ & 0,33 & $-0,52$ & 0,54 & 2.432 \\
\hline $\mathrm{NG}^{\prime}+40 \%+\mathrm{Cal}$ & 14,2 & 1,870 & 84 & $32.806,5$ & $-0,27$ & $-0,18$ & 0,65 & 3.030 \\
\hline $\mathrm{NG}^{\prime}+60 \%+\mathrm{Cal}$ & 13,3 & 1,975 & 84 & $76.420,9$ & $-0,07$ & $-0,65$ & 0,87 & 1.438 \\
\hline $\mathrm{NG}^{\prime}+70 \%+\mathrm{Cal}$ & 12,4 & 2,030 & 84 & $46.756,8$ & $-0,41$ & $-0,27$ & 0,87 & 1.380 \\
\hline
\end{tabular}
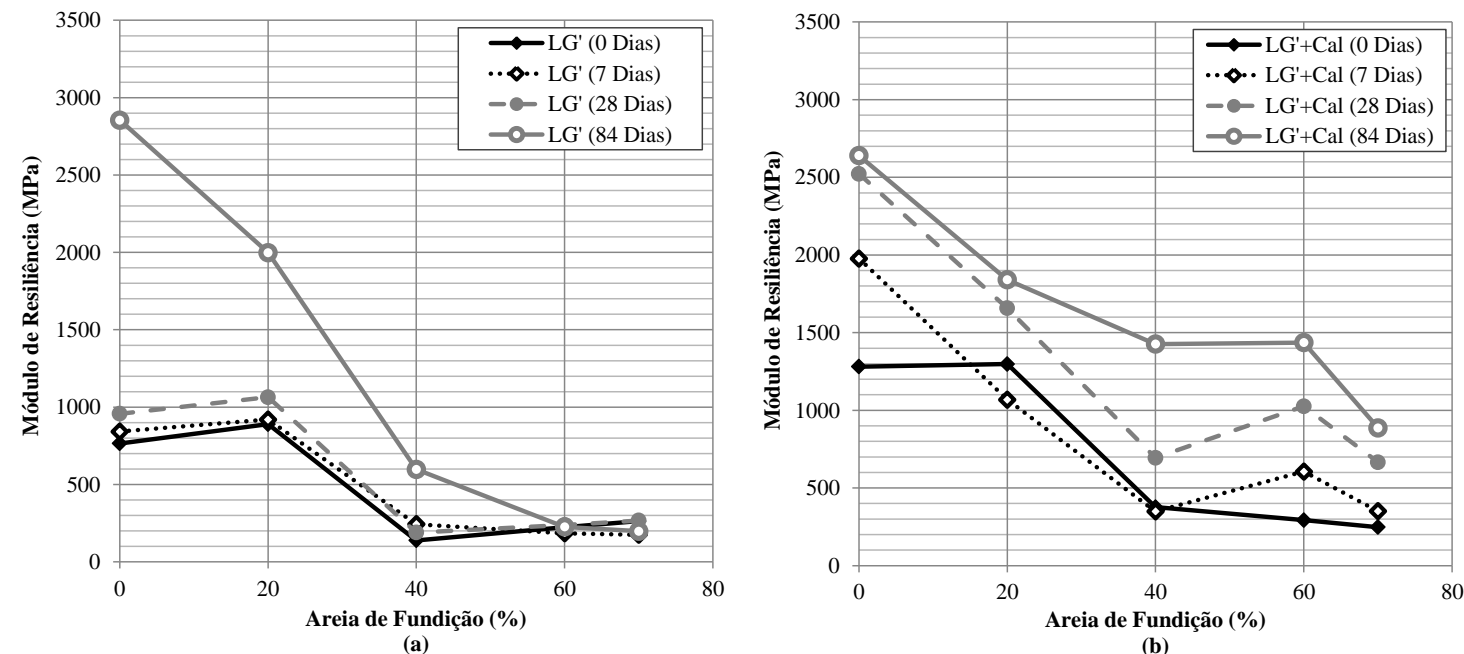

Figura 3 - Módulo de resiliência das misturas com solo LG', para o par de tensões ( $\sigma_{3}=137,9 \mathrm{kPa}$ e $\sigma_{\mathrm{d}}=275,8 \mathrm{kPa}$ ), sem (a) e com cal (b) 


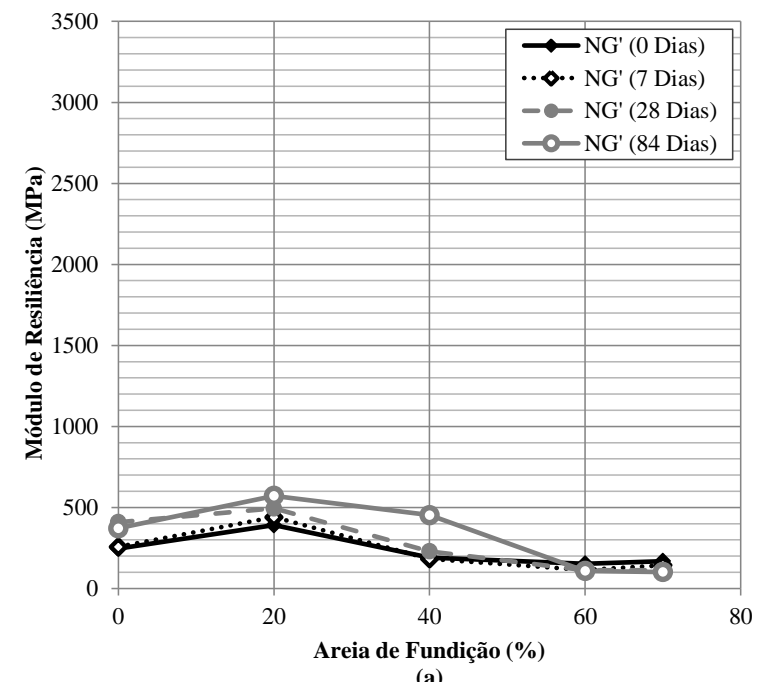

(a)

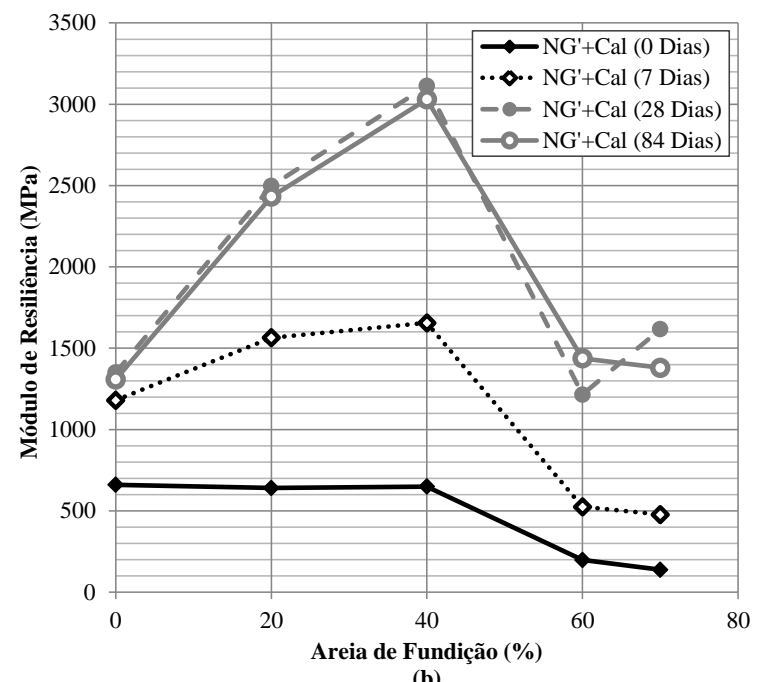

(b)

Figura 4 - Módulo de resiliência das misturas com solo NG', para o par de tensões $\left(\sigma_{3}=137,9 \mathrm{kPa}\right.$ e $\left.\sigma_{\mathrm{d}}=275,8 \mathrm{kPa}\right)$, sem (a) e com cal (b)

\subsection{Adição de Areia de Fundição Residual}

$\mathrm{O}$ incremento do teor de areia de fundição residual nas misturas compostas pelo solo LG' reduziu os valores de Módulo de Resiliência. Esse comportamento é notado tanto nas misturas Solo LG'+AFR como nas misturas Solo LG' $+\mathrm{AFR}+\mathrm{Cal}$.

Nas misturas Solo NG'+AFR, os maiores valores de módulo foram observados nos solos com adição de $20 \%$ do resíduo, para todos os tempos de cura, como notado na Figura 4a. Já nas misturas Solo NG'+AFR+Cal (Figura 4b), a adição de $40 \%$ de areia incrementou os valores de $M_{R}$, aos 7, 28 e 84 dias de cura.

\subsection{Adição de Cal Hidratada}

A adição de $3 \%$ de cal hidratada nas misturas compostas pelo solo LG' incrementou os valores de Módulo de Resiliência, para todos os tempos de cura.

As misturas Solo NG'+AFR+Cal apresentaram valores de $M_{R}$ bastante superiores, quando comparadas com as misturas Solo $\mathrm{NG}^{\prime}+\mathrm{AFR}$. Nota-se na Figura $4 \mathrm{~b}$ um notável incremento aos 28 e 84 dias de cura.

Ainda pode se observar nas Figuras 3 e 4 que as misturas Solo LG'+AFR apresentam valores de módulo maiores que as misturas Solo NG'+AFR. Contudo, as misturas Solo $\mathrm{NG}+\mathrm{AFR}+\mathrm{Cal}$ apresentaram $M_{R}$ mais elevados que as misturas LG'+AFR+Cal. Esse fenômeno pode indicar uma maior reatividade do solo $\mathrm{NG}^{\prime}$ à adição de cal hidratada.

\subsection{Resultados dos Ensaios Ambientais}

A Tabela 5 apresenta os resultados de classificação de acordo segundo a ABNT, NBR 10004:2006. Nessa Tabela nota-se que a Areia de Fundição Residual é classificada como um Resíduo Não Perigoso e Não Inerte, Classe II-A. Também observa-se que os solos puros, LG' e NG' são classificados como Resíduos Classe IIA.

\begin{tabular}{|c|c|}
\hline Material & Classificação (NBR 10004:2006) \\
\hline Areia de Fundição Residual (AFR) & Classe II-A \\
\hline Solo Argiloso Laterítico (LG') & Classe II-A \\
\hline Solo Argiloso Não Laterítico (NG') & Classe II-A \\
\hline LG' $^{\prime}+60 \%$ AFR & Classe II-A \\
\hline$N G^{\prime}+60 \%$ AFR & Classe II-A \\
\hline $\mathrm{LG}^{\prime}+60 \% \mathrm{AFR}+3 \% \mathrm{Cal}$ & Classe II-A \\
\hline $\mathrm{NG}^{\prime}+60 \% \mathrm{AFR}+3 \% \mathrm{Cal}$ & Classe II-A \\
\hline
\end{tabular}


Ainda na Tabela 5 é notado que a adição de $60 \%$ de AFR nos solos LG' e NG' não modificou a classificação de Resíduo Não Perigoso e Não Inerte, Classe II-A. A adição de 3\% de cal hidratada tampouco modificou essa classificação. Assim pode-se afirmar que o emprego da areia de fundição residual em misturas com os solos argilosos utilizados nesta pesquisa não representam risco de poluição para o meio ambiente.

\section{CONCLUSÕES}

Este artigo estudou a influência no módulo de resiliência da adição de areia de fundição residual e cal hidratada em solos argilosos. Também foram avaliadas cinco equações matemáticas utilizadas para modelar os valores do $M_{R}$ desses materiais obtidos dos ensaios triaxiais cíclicos. Dos resultados pode-se concluir:

- o Modelo Composto é o que apresenta melhor ajuste para os resultados dos ensaios de determinação do módulo de resiliência, já que foi o que apresentou maior porcentagem de regressões com $\mathrm{R}^{2} \geq 0,90$;

- o tempo de cura influencia diretamente no valor de $M R$ e esta influência é mais notória para tempos de cura elevados nas misturas que contém cal hidratada;

- a adição de areia de fundição residual reduziu os valores de módulo nas misturas compostas pelo solo LG'. Já nas composições NG'+AFR com $20 \%$ de areia de fundição e a $\mathrm{NG}^{\prime}+\mathrm{AFR}+\mathrm{Cal}$ com $40 \%$ houve aumento dos valores de Módulo de Resiliência;

- a presença de $3 \%$ de cal hidratada nas misturas aumentou os valores de $M_{R}$, principalmente para tempos de cura elevados. Notou-se também que a adição de cal proporcionou aumentos maiores dos módulos nas misturas com solo $\mathrm{NG}^{\prime}$ do que nas com o LG', provavelmente devido à maior reatividade do solo NG' à cal hidratada.

De modo geral, as misturas apresentaram valores de módulo de resiliência semelhantes e até superiores aos usualmente obtidos em materiais mais nobres, tais como a brita graduada, o solo-brita e o solo arenoso fino laterítico, comumen- te utilizados como materiais de construção de camadas de pavimentos. Contudo, vale ressaltar que o valor do Módulo de Resiliência deve ser analisado em conjunto com outros parâmetros que consideram o comportamento do material face aos efeitos da secagem e do umedecimento.

Em relação aos ensaios ambientais, a AFR foi classificada como um Resíduo Não Perigoso e Não Inerte, (Classe II-A), e sua incorporação aos solos LG' e NG', com ou sem cal hidratada, não modificou essa classificação. Assim, pode-se concluir que o reaproveitamento de até $60 \%$ de areia de fundição residual em misturas com solos argilosos, não representa um risco de poluição ao meio ambiente.

\section{REFERÊNCIAS}

AASHTO T 307-99 (1999).Standard Method of Test for determining the resilient Modulus of Soils and Aggregate Materials. American Association of State Highway and Transportation Officials.

AASHTO (2002). Guide for Mechanistic-Empirical Design of new and rehabilitated pavement structures. http://onlinepubs.trb.org

Andrei, D., M. W. Witczak, C. W. Schwartz, J. Uzan. (2004). Harmonized Resilient modulus test method for unbound pavement materials. Transportation Research Record. 1874, Transportation Research Board, Washington, D.C., pp. 29-37. (DOI: 10.3141/1874-04)

Baptista, C. F. N. (1976). Pavimentação, Compactação dos solos no campo, camadas de base, estabilização dos solos. Livro. Tomo II. Editora Globo. Porto Alegre, RS

Boudreau, R. L. e Wang, J. (2003). Resilient modulus test-Triaxial cell interaction. Resilient modulus testing for pavement components, ASTM STP 1437, G. N. Durham, W. A. Marr, and W. L. De Groff, Eds., ASTM International, West Conshohocken, PA.

Dunlap, W. S. (1963). A report on a mathematical model describing the deformation characteristics of granular materials. Technical Report 1, Project 2-8-62-27, TTI, Texas A \& M University.

Ferreira, J. G. H. M. (2002). Elaboração e análise da base de dados de ensaios triaxiais dinâmicos da COPPE/UFRJ. Dissertação (Mestrado) - PEC, COPPE/UFRJ, Rio de Janeiro.

Jung, C.; Bobet, A.; Siddiki, N. Z.; Kim, D. (2008). Long-Term Performance of Chemically Modified Subgrade Soils in Indiana. Transportation Research Record: Journal of the Transportation Research Board, No. 2029, pp. 63-71. Washington D.C. United States. (DOI: 10.3141/2059-07)

Karasahin, M., Dawnson, A. R., Holden, J.T. (1993). Applicability of resilient constitutive models of granular materials for unbound base layers. Transportation Research Record. 1406, Transportation Research Board, Washington, D.C., pp. 98-107.

Klinsky, L. M. G. (2008). Proposta de Reaproveitamento de areia de fundição em sub-bases e bases de pavimentos flexíveis, através de sua incorporação a solos argilosos. Dissertação (Mestrado) - Escola de Engenharia de São Carlos - Universidade de São Paulo. São Paulo. 
Leandro, R. P. (2005). Estudo laboratorial acerca da possibilidade de aproveitamento da cinza pesada de termelétrica em bases e sub-bases de pavimentos flexíveis. Dissertação (Mestrado). Escola de Engenharia de São Carlos - Universidade de São Paulo. São Paulo.

Little, N. D. (1999). Evaluation of structural properties of lime stabilized soils and aggregates. Volume 1: Summary of findings. National Lime Association. Estados Unidos da América.

Lovato, R. S. (2004). Estudo do Comportamento Mecânico de um Solo Laterítico Estabilizado com Cal, Aplicado à Pavimentação. Dissertação (Mestrado). Universidade Federal do Rio Grande do Sul. Porto Alegre - Rio Grande do Sul.

Medina, J. De; Motta, L. M. G. Da. (2005). Mecânica dos Pavimentos. Livro, $2^{\text {a }}$ Edição. Rio de Janeiro.

Moosazadeh, J. M., and Witczak, W. (1981). Prediction of subgrade moduli for soil that exhibits nonlinear behavior. Transportation Research Record. Transportation Research Board, National Research Council Washington, D.C.
NCHRP. (1997) Laboratory determination of resilient modulus for flexible pavement design. NCHRP Web Document 14 for Project 1-28. Transportation Research Board, Washington, D.C.

Oliveira, S.M.F. (2005). Estudo do comportamento mecânico de misturas de fosfogesso e cal para utilização na construção rodoviária. Dissertação (Mestrado) - Escola de Engenharia de São Carlos - Universidade de São Paulo. São Paulo.

Pan, T.Y., Tutumluer, E., Anochie-Boateng, J. (2006). Aggregate Morphology affecting resilient behavior of unbound granular materials. Transportation Research Record. 1952, Transportation Research Board, Washington, D.C., pp. 12-20. (DOI: 10.3141/195202)

Solanki, P.; Zaman, M. M.; Dean, J. (2010) Resilient Modulus of Clay Subgrades Stabilized with Lime, Class C Fly Ash and Cement Kiln Dust for Pavement Design. Transportation Research Record: Journal of the Transportation Research Board, No. 2186. (DOI: $10.3141 / 2186-11$ ) 